\title{
Local Existence for the One-dimensional Vlasov-Poisson System with Infinite Mass
}

\author{
Stephen Pankavich \\ Department of Mathematics \\ Indiana University \\ Bloomington, IN 47401 \\ sdp@indiana.edu
}

November 20, 2018

Mathematics Subject Classification : 35L60, 35Q99, 82C21, 82C22, 82D10.

\begin{abstract}
A collisionless plasma is modelled by the Vlasov-Poisson system in one dimension. We consider the situation in which mobile negative ions balance a fixed background of positive charge, which is independent of space and time, as $|x| \rightarrow \infty$. Thus, the total positive charge and the total negative charge are both infinite. Smooth solutions with appropriate asymptotic behavior are shown to exist locally in time, and criteria for the continuation of these solutions are established.
\end{abstract}

\section{Introduction}

Consider the following simplified model of a collisionless plasma, using the Vlasov-Poisson system in one dimension. Let $F: \mathbb{R} \rightarrow[0, \infty)$ and $f_{0}: \mathbb{R} \times \mathbb{R} \rightarrow[0, \infty)$ be given, and seek a function $f:[0, \infty) \times \mathbb{R} \times \mathbb{R} \rightarrow[0, \infty)$ such that

$$
\begin{gathered}
\partial_{t} f+v \partial_{x} f-E \partial_{v} f=0 \\
\rho(t, x)=\int(F(v)-f(t, x, v)) d v \\
E(t, x)=\frac{1}{2}\left(\int_{-\infty}^{x} \rho(t, y) d y-\int_{x}^{\infty} \rho(t, y) d v\right) \\
f(0, x, v)=f_{0}(x, v)
\end{gathered}
$$

where $t \in[0, \infty)$ denotes time, $x \in \mathbb{R}$ denotes space, and $v \in \mathbb{R}$ denotes momentum. Here, $f$ gives the density in phase space of mobile negative ions, while $F$ describes a number density of positive ions which form a fixed background. We seek solutions for which $f(t, x, v) \rightarrow F(v)$ as $|x| \rightarrow \infty$. Precise conditions which ensure local-in-time existence of such solutions to (11) are given in Section 1. 
To this end, we wish to proceed in a manner similar to [13, which proved the existence of a localin-time solution to the three dimensional analogue of (11). In that paper, the existence argument hinged upon showing that $\rho$ decayed faster than $|x|^{-3}$. In the same vein, the main difficulty of this paper arises in showing $\rho$ decays rapidly enough in $|x|$, in this case, better than $|x|^{-1}$. A crucial difference between these arguments, however, lies in the decay rates of the other functions. Let $g=F-f$. In the three dimensional problem it is well known that $E, g$ and $\nabla g$ decay at a rate of $|x|^{-2}$. For (11), none of the analogous terms are initially known to tend to zero for large $x$. Indeed, of these terms only $\partial_{x} g$ can be shown to do so. One important part of the existence proof in [13] relied upon Lemma 1 and 5 of that paper generating the estimate :

$$
\rho \approx \int E \cdot \nabla g d v \approx|x|^{-4}
$$

Due to the differing behavior of $E$ and $\nabla g$ in (10), this estimate does not hold in the one dimensional problem. Instead, since neither $E$ nor $\nabla g$ are known to decay in space, it is unclear as to the a priori behavior of $\rho$. This difficulty is remedied through the use of Lemma 4. Regardless, it is worth noting that due to a loss of spatial decay in each function, (1) contains additional difficulties to those encountered in the three dimensional problem, and many of the techniques used in [13. cannot be utilized here.

The Vlasov-Poisson system has been studied extensively in the case where $F(v)=0$ and solutions tend to zero as $|x| \rightarrow \infty$, mostly with respect to the three-dimensional problem. Most of the literature involving the one-dimensional Vlasov-Poisson system focus on time asymptotics, such as [2] and [3]. Much more work has been done concerning the three-dimensional problem. Smooth solutions were shown to exist globally-in-time in [9] (refined in [11]) and independently in [6]. Important results preliminary to the discovery of a global-in-time solution include [1] and [5]. A complete discussion of the literature concerning the Vlasov-Poisson system may be found in [4], and more recently [10].

A good deal of progress has also been made on the infinite mass problem for the Vlasov-Poisson system, this being the three-dimensional analogue of (1). Other than [13, smooth solutions were shown to exist globally-in-time for the case of a radial field in [7]. A priori bounds on the charge and current densities were established in [12], assuming $F(v)$ is suitably smooth, radial and decreasing. Finally, using the bounds derived in [12, a unique global-in-time smooth solution was shown to exist in [8] for an arbitrary electric field, assuming $F$ is compactly supported. Hence, our assumptions on data will mimic that of [13] and [8].

\section{Section 1}

We let $p>1$ be given and use the following notation. Denote

$$
R(x)=R(|x|)=\sqrt{1+|x|^{2}} .
$$

For given functions $h: \mathbb{R}^{2} \rightarrow \mathbb{R}$ and $\sigma: \mathbb{R} \rightarrow \mathbb{R}$, we will use the norms

$$
\begin{gathered}
\|h\|_{\infty}=\sup _{z \in \mathbb{R}^{2}}|h(z)|, \\
\|\sigma\|_{p}=\left\|\sigma R^{p}(x)\right\|_{L^{\infty}(\mathbb{R})},
\end{gathered}
$$


and

$$
\||h|\|=\|h\|_{\infty}+\left\|\partial_{v} h\right\|_{\infty}+\left\|\partial_{x} h\right\|_{p}+\left\|\int h d v\right\|_{p}
$$

but never use the $L^{p}$ norm (for $p$ finite). For example, we will write $\|\rho(t)\|_{p}$ for the $\|\cdot\|_{p}$ norm of $x \rightarrow \rho(t, x)$.

In order to study the above system, we will assume the following conditions hold throughout : (A-1) $f_{0} \in \mathcal{C}^{1}\left(\mathbb{R}^{2}\right)$ is nonnegative with compact $v$-support and for $x, v \in \mathbb{R}$

$$
\left|\left(F-f_{0}\right)(x, v)\right|+\left|\partial_{v}\left(F-f_{0}\right)(x, v)\right| \leq C R^{-p}(x) .
$$

(A-2) $F \in \mathcal{C}^{3}(\mathbb{R})$ is nonnegative and there is $W \in(0, \infty)$ such that for $|v|>W$,

$$
F(v)=0 .
$$

(A-3) $\left\|\left|\left(F-f_{0}\right)\right|\right\|$ is finite.

From these assumptions, local-in-time existence follows.

Theorem 1 Assuming (A-1) thru (A-3) hold, there exist $\delta>0$ and $f \in C^{1}\left([0, \delta] \times \mathbb{R}^{2}\right)$ satisfying (11) with $\||(F-f)(t)|\| \leq C$ for $t \in[0, \delta]$. Moreover, $f$ is unique.

In addition, we may continue the local-in-time solution as long as this norm remains bounded.

Theorem 2 Assume (A-1) thru (A-3) hold. Let $T>0$ be given and $f$ be a $C^{1}$ solution of (1) on $[0, T] \times \mathbb{R}^{2}$. If

$$
\||(F-f)(t)|\| \leq C
$$

on $[0, T]$, then we may extend the solution to $[0, T+\delta]$ for some $\delta>0$ where $\||(F-f)(t)|\|$ is bounded on $[0, T+\delta]$.

In order to combine the proofs of Theorems 1 and 2 , let $f^{(T)}=f_{0}$ if $T=0$ and for $T>0$, let $f^{(T)}$ be the solution assumed to exist in Theorem 2. For $\delta>0$ and $r>1$, define

$$
\begin{aligned}
\mathcal{C}=\mathcal{C}(\delta, r):= & \left\{f \in C^{1}\left([0, T+\delta] \times \mathbb{R}^{2}\right):\right. \\
& f(t, x, v)=f^{(T)}(t, x, v) \text { if } t \in[0, T] \\
& \text { and }\|\mid(F-f)(t)\| \leq r \text { if } t \in[0, T+\delta]\} .
\end{aligned}
$$

We take

$$
r \geq 1+\sup _{t \in[0, T]}\||(F-f)(t)|\|
$$

and $0 \leq \delta \leq \frac{1}{r}$ for the remainder of the paper.

For $f \in \mathcal{C}$, define

$$
\begin{aligned}
g & =F-f \\
\rho & =\int g d v \\
E & =\frac{1}{2}\left(\int_{-\infty}^{x} \rho(t, y) d y-\int_{x}^{\infty} \rho(t, y) d y\right) .
\end{aligned}
$$


Further define $\mathcal{F}[f]=\tilde{f}$ by

$$
\left\{\begin{aligned}
\tilde{f}(t, x, v)=f^{(T)}(t, x, v), & t \in[0, T] \\
\partial_{t} \tilde{f}+v \partial_{x} \tilde{f}-E \partial_{v} \tilde{f}=0, & t \in[T, T+\delta]
\end{aligned}\right.
$$

Then,

$$
\tilde{g}=F-\tilde{f}
$$

and

$$
\tilde{\rho}=\int \tilde{g} d v
$$

In Section 2, we will choose $r$ and $\delta$ such that $\mathcal{F}: \mathcal{C} \rightarrow \mathcal{C}$, and use this to show that an iterative sequence converges, thus proving Theorems 1 and 2. Unless it is stated otherwise, we will denote by "C" a generic constant which changes from line to line and may depend upon $f_{0}, F, T$, or $\sup _{t \in[0, T]}\||(F-f)(t)|\|$, but not on $t, x, v, r$ or $\delta$. When it is necessary to refer to specific constants, we will use superscripts. For instance, $C^{(1)}$ will always denote the same value.

Define

$$
I(t-T)= \begin{cases}0, & t \leq T \\ 1, & t>T\end{cases}
$$

Then, by definition, $f \in \mathcal{C}$ implies

$$
\|\rho(t)\|_{p} \leq C(1+r I(t-T))
$$

for all $x \in \mathbb{R}$ and $t \in[0, T+\delta]$. Therefore, given $t \in[0, T+\delta]$ and $x \in \mathbb{R}$, we find

$$
|E(t, x)| \leq\|\rho(t)\|_{p}\left(\int R^{-p}(y) d y\right) \leq C\|\rho(t)\|_{p} .
$$

Thus, for $t \in[0, T+\delta]$, we find

$$
\int_{0}^{t}\|E(s)\|_{\infty} d s \leq C \int_{0}^{t}(1+r I(s-T)) d s \leq C(1+r \delta) \leq C=: C^{(1)}
$$

and the field integral is uniformly bounded. Define the characteristics, $X(s, t, x, v)$ and $V(s, t, x, v)$, as solutions to the system of ordinary differential equations :

$$
\left.\begin{array}{c}
\frac{\partial}{\partial s} X(s, t, x, v)=V(s, t, x, v) \\
\frac{\partial}{\partial s} V(s, t, x, v)=-E(s, X(s, t, x, v)) \\
X(t, t, x, v)=x \\
V(t, t, x, v)=v .
\end{array}\right\}
$$

We will make use of the following lemma which results merely from this bound on the time integral of the field.

Lemma 1 Let $t \in[0, T+\delta], s \in[0, t]$, and $x \in \mathbb{R}$ be given. Then, for any $v \in \mathbb{R}$,

$$
|v|-C^{(1)} \leq|V(s, t, x, v)| \leq|v|+C^{(1)} .
$$

In particular, for $|v|>2 C^{(1)}$,

$$
\frac{1}{2}|v| \leq|V(s, t, x, v)| \leq \frac{3}{2}|v| .
$$


The following lemma is the crucial tool used in showing decay (at a rate of $|x|^{-p}$ ) of the charge density, $\rho$.

Lemma 2 Assume that $\mathcal{E}: \mathbb{R} \rightarrow \mathbb{R}$ is $\mathcal{C}^{1}$ and there exists $B>0$ such that

$$
|\mathcal{E}(x)| \leq B
$$

and

$$
\left|\mathcal{E}^{\prime}(x)\right| \leq B R^{-p}(x)
$$

for all $x \in \mathbb{R}$. Also, assume $H: \mathbb{R} \rightarrow \mathbb{R}$ is $\mathcal{C}_{c}^{2}$. Then,

$$
\left|\int \mathcal{E}(X(s, t, x, v)) H^{\prime}(V(s, t, x, v)) d v\right| \leq C B R^{-p}(x)
$$

for all $x \in \mathbb{R}$ and $0 \leq s \leq t \leq T+\delta$.

We will postpone the proofs of all lemmas until Section 3.

\section{Section 2}

Let us estimate $\|\tilde{\rho}(t)\|_{p}$. Define

$$
D=\partial_{t}+v \partial_{x}-E \partial_{v}
$$

Then,

$$
D \tilde{g}=-E F^{\prime}
$$

so that

$$
\tilde{\rho}(t, x)=\rho_{0}(t, x)-\int_{0}^{t} \int E(s, X(s, t, x, v)) F^{\prime}(V(s, t, x, v)) d v d s
$$

where

$$
\rho_{0}(t, x)=\int\left(F-f_{0}\right)(X(0, t, x, v), V(0, t, x, v)) d v .
$$

Since $\partial_{x} E=\rho$, we use (6) and (77) in Lemma 2 to find

$$
\begin{aligned}
\left|\int_{0}^{t} \int E(s, X(s, t, x, v)) F^{\prime}(V(s, t, x, v)) d v d s\right| & \leq \int_{0}^{t} C(1+r I(s-T)) R^{-p}(x) d s \\
& \leq C(1+r \delta) R^{-p}(x) \\
& \leq C R^{-p}(x) .
\end{aligned}
$$

Then, to estimate $\rho_{0}$, we use (A-1) and (A-2) to conclude that $F-f_{0}$ has compact $v$-support. Let $P_{V}:=\sup \left\{|v|: \exists x \in \mathbb{R}\right.$ such that $\left.\left(F-f_{0}\right)(x, v) \neq 0\right\}$ and define $P_{v}:=P_{V}+C^{(1)}$. Using Lemma 1 for $|v|>P_{v}$, we find $|V(0)|>P_{V}$ and thus $\left(F-f_{0}\right)(X(0), V(0))=0$. It follows that $\left(F-f_{0}\right)(X(0), V(0))$ has compact support as a function of $v$. Now, assume $|v| \leq P_{v}$. For $|x|>2\left(P_{v}+C^{(1)}\right)(T+\delta)$, we again use Lemma 1 to find

$$
|X(0, t, x, v)| \geq|x|-\int_{0}^{t}|V(\tau)| d \tau \geq|x|-\left(P_{v}+C^{(1)}\right)(T+\delta) \geq \frac{1}{2}|x|
$$


and thus $R^{-p}(X(0)) \leq R^{-p}\left(\frac{1}{2}|x|\right) \leq C R^{-p}(x)$. In addition, for $|x| \leq 2\left(P_{v}+C^{(1)}\right)(T+\delta)$, we have $R^{-p}(X(0)) \leq C \leq C R^{-p}(x)$. Therefore, we find for any $x \in \mathbb{R}$,

$$
R^{-p}(X(0)) \leq C R^{-p}(x) .
$$

Hence, we find

$$
\begin{aligned}
\left|\rho_{0}(t, x)\right| & \leq \int_{|v| \leq P_{v}} C R^{-p}(X(0, t, x, v)) d v \\
& \leq C R^{-p}(x) .
\end{aligned}
$$

Using this and (13), we find

$$
|\tilde{\rho}(t, x)| \leq C R^{-p}(x)
$$

and thus

$$
\|\tilde{\rho}(t)\|_{p} \leq C .
$$

Now, we estimate $\tilde{g}, \partial_{v} \tilde{g}$, and $\partial_{x} \tilde{g}$. First, we use (A-2), (A-3), and (8) in (11), after integrating along characteristics, to find

$$
\begin{aligned}
|\tilde{g}(t, x, v)| & \leq\left|\left(F-f_{0}\right)(X(0), V(0))\right|+\int_{0}^{t}|E(s, X(s))|\left|F^{\prime}(V(s))\right| d s \\
& \leq C+\left\|F^{\prime}\right\|_{\infty}\left(\int_{0}^{t}|E(s, X(s))| d s\right) \\
& \leq C .
\end{aligned}
$$

Using $D$ defined by (10), we have

$$
D\left(\partial_{x} \tilde{g}\right)=\partial_{x} E\left(\partial_{v} \tilde{g}-F^{\prime}\right)=\rho\left(\partial_{v} \tilde{g}-F^{\prime}\right)
$$

and

$$
D\left(\partial_{v} \tilde{g}\right)=-\left(\partial_{x} g+E F^{\prime \prime}\right) .
$$

Then, using (6), (7), and (A-2), we find

$$
\left|D\left(\partial_{x} \tilde{g}\right)\right| \leq C R^{-p}(x)(1+r I(t-T))\left(1+\left|\partial_{v} \tilde{g}\right|\right)
$$

and

$$
\left|D\left(\partial_{v} \tilde{g}\right)\right| \leq C(1+r I(t-T))\left(1+\left|\partial_{x} \tilde{g}\right|\right)
$$

Denoting

$$
\tilde{G}(s)=\left(\left|\partial_{v} \tilde{g}\right|+\left|\partial_{x} \tilde{g}\right|\right)(s, X(s), V(s)),
$$

we combine (16) and (17) and integrate along characteristics to find

$$
\tilde{G}(t) \leq \tilde{G}(0)+C \int_{0}^{t}(1+r I(s-T))(1+\tilde{G}(s)) d s .
$$

By Gronwall's inequality and (A-3), we find

$$
\tilde{G}(t) \leq C(1+\tilde{G}(0)) \leq C,
$$

and it follows that for any $t \in[0, t+\delta]$,

$$
\|\tilde{g}(t)\|_{\infty}+\left\|\partial_{v} \tilde{g}(t)\right\|_{\infty}+\left\|\partial_{x} \tilde{g}(t)\right\|_{\infty} \leq C .
$$

For the remainder of the section, we will need to estimate terms which involve $v$-integrals of $\tilde{g}$. Thus, the following lemma will be useful : 
Lemma 3 For any $f \in \mathcal{C}$, define $\tilde{f}$ by (5) and $\tilde{g}=F-\tilde{f}$. Then, $Q_{\tilde{g}}(t)$ defined by

$$
Q_{\tilde{g}}(t)=\sup \{|v|: \exists x \in \mathbb{R}, \tau \in[0, t] \text { such that } \tilde{g}(\tau, x, v) \neq 0\} .
$$

is bounded for all $t \in[0, T+\delta]$.

Continuing with the estimate of $\||\tilde{g}|\|$, we use (16) and (18), and integrate along characteristics to find

$$
\left|\partial_{x} \tilde{g}(t, x, v)\right| \leq \mid \partial_{x} \tilde{g}\left(0, X(0), V(0) \mid+C \int_{0}^{t}(1+r I(s-T)) R^{-p}(X(s)) d s .\right.
$$

By the definition of $Q_{\tilde{g}}(t)$, we find that for $|x|>2(T+\delta) Q_{\tilde{g}}(T+\delta)$, we have

$$
|X(s, t, x, v)| \geq|x|-\int_{s}^{t}|V(\tau)| d \tau \geq|x|-(T+\delta) Q_{\tilde{g}}(T+\delta) \geq \frac{1}{2}|x|
$$

and thus $R^{-p}(X(s)) \leq R^{-p}\left(\frac{1}{2}|x|\right) \leq C R^{-p}(x)$. In addition, for $|x| \leq 2(T+\delta) Q_{g}(T+\delta)$, we have $R^{-p}(X(s)) \leq C \leq C R^{-p}(x)$. Therefore, we find for any $x \in \mathbb{R}$,

$$
R^{-p}(X(s)) \leq C R^{-p}(x) .
$$

We use this and (A-3) to find

$$
\left|\partial_{x} \tilde{g}(t, x, v)\right| \leq C R^{-p}(x)
$$

and therefore

$$
\left\|\partial_{x} \tilde{g}(t, x, v)\right\|_{p} \leq C .
$$

Collecting (14), (18), and (20) we find

$$
\||\tilde{g}(t, x, v)|\| \leq C^{(2)} .
$$

We take $r \geq C^{(2)}$ and conclude that $\tilde{f} \in \mathcal{C}$ and $\mathcal{F}: \mathcal{C} \rightarrow \mathcal{C}$.

Next, let $f, \rho, E, \tilde{f}$, and $\tilde{\rho}$ be as before and for $h \in \mathcal{C}$, define

$$
\begin{aligned}
& g_{h}=F-h, \\
& \rho_{h}=\int g_{h} d v \\
& E_{h}=\frac{1}{2}\left(\int_{-\infty}^{x} \rho_{h}(t, y) d y-\int_{x}^{\infty} \rho_{h}(t, y) d y\right), \\
& \tilde{h}=\mathcal{F}[h], \\
& \tilde{g}_{h}=F-\tilde{h},
\end{aligned}
$$

and

$$
\tilde{\rho}_{h}=\int \tilde{g}_{h} d v
$$

We estimate $\left\|\tilde{\rho}(t)-\tilde{\rho}_{h}(t)\right\|_{p}$ so that we may apply a uniform bound to the iterates defined later. As before, we consider $D$ defined by (10) and find

$$
D(\tilde{f}-\tilde{h})=\left(E-E_{h}\right) \partial_{v} \tilde{h}=\left(E-E_{h}\right)\left(F^{\prime}-\partial_{v} \tilde{g}_{h}\right) .
$$


So, we have

$$
\begin{aligned}
\left(\tilde{\rho}-\tilde{\rho}_{h}\right)(t, x)= & \int_{0}^{t} \int\left(E-E_{h}\right)(s, X(s)) F^{\prime}(V(s)) d v d s \\
& -\int_{0}^{t} \int\left(E-E_{h}\right)(s, X(s)) \partial_{v} \tilde{g}_{h}(s, X(s), V(s)) d v d s
\end{aligned}
$$

where $X(s)$ and $V(s)$ are defined by (9). Since $f, h \in \mathcal{C}$, we use (4) and (7) to find

$$
\left|\left(E-E_{h}\right)(t, x)\right| \leq C\left\|\rho(t)-\rho_{h}(t)\right\|_{p}
$$

and

$$
\left|\partial_{x}\left(E-E_{h}\right)(t, x)\right| \leq C\left\|\rho(t)-\rho_{h}(t)\right\|_{p} R^{-p}(x) .
$$

Using Lemma 2, we have

$$
\left|\int\left(E-E_{h}\right)(s, X(s)) F^{\prime}(V(s)) d v\right| \leq C\left\|\left(\rho-\rho_{h}\right)(s)\right\|_{p} R^{-p}(x) .
$$

Thus, it follows that

$$
\left.\left|\int_{0}^{t} \int\left(E-E_{h}\right)(s, X(s)) F^{\prime}(V(s)) d v d s\right| \leq C\left(\int_{0}^{t} \| \rho-\rho_{h}\right)(s) \|_{p} d s\right) R^{-p}(x) .
$$

To estimate the remaining portion of (22), we will use :

Lemma 4 For $0 \leq s \leq t \leq T+\delta$ and $x \in \mathbb{R}$,

$$
\left|\int\left(E-E_{h}\right)(s, X(s)) \partial_{v} \tilde{g}_{h}(s, X(s), V(s)) d v\right| \leq C\left\|\left(\rho-\rho_{h}\right)(s)\right\|_{p} R^{-p}(x) .
$$

It follows from Lemma 4 that

$$
\left|\int_{0}^{t} \int\left(E-E_{h}\right)(s, X(s)) \partial_{v} \tilde{g}_{h}(s, X(s), V(s)) d v d s\right| \leq C\left(\int_{0}^{t}\left\|\left(\rho-\rho_{h}\right)(s)\right\|_{p} d s\right) R^{-p}(x) .
$$

Using (23) and (24) in (22), we find

$$
\left\|\left(\tilde{\rho}-\tilde{\rho}_{h}\right)(t)\right\|_{p} \leq C^{(3)}\left(\int_{0}^{t}\left\|\left(\rho-\rho_{h}\right)(s)\right\|_{p} d s\right) .
$$

Now, define the first iterate by

$$
f^{(0)}(t, x, v)= \begin{cases}f^{(T)}(t, x, v) & \text { if } t \in[0, T] \\ f^{(T)}(T, x, v) & \text { if } t \in[T, T+\delta] .\end{cases}
$$

Then, for $k=0,1,2,3, \ldots$, assuming $f^{(k)}$ is known, define

$$
\begin{aligned}
g^{(k)} & =F-f^{(k)} \\
\rho^{(k)} & =\int g^{(k)} d v \\
E^{(k)} & =\frac{1}{2}\left(\int_{-\infty}^{x} \rho^{(k)}(t, y) d y-\int_{x}^{\infty} \rho^{(k)}(t, y) d y\right), \\
f^{(k+1)} & =\mathcal{F}\left[f^{(k)}\right] .
\end{aligned}
$$


Since $f^{(0)}, f^{(1)} \in \mathcal{C}$, we have

$$
\left\|\left(\rho^{(1)}-\rho^{(0)}\right)(t)\right\|_{p} \leq C^{(4)} I(t-T) .
$$

Then, we use (25) to find

$$
\left\|\left(\rho^{(2)}-\rho^{(1)}\right)(t)\right\|_{p} \leq C^{(3)} C^{(4)}(t-T) I(t-T),
$$

and

$$
\left\|\left(\rho^{(3)}-\rho^{(2)}\right)(t)\right\|_{p} \leq C^{(4)} \frac{1}{2}\left(C^{(3)}(t-T)\right)^{2} I(t-T) .
$$

This can be repeated and using induction, for any $k=0,1,2,3, \ldots$, we have

$$
\left\|\left(\rho^{(k+1)}-\rho^{(k)}\right)(t)\right\|_{p} \leq \frac{C^{(4)}}{k !}\left(C^{(3)}(t-T)\right)^{k} I(t-T) .
$$

Finally, for $m, n \in \mathbb{Z}^{+}$with $m>n$, we have

$$
\begin{aligned}
\left\|\left(\rho^{(m)}-\rho^{(n)}\right)(t)\right\|_{p} & \leq \sum_{\ell=n}^{m-1}\left\|\left(\rho^{(\ell+1)}-\rho^{(\ell)}\right)(t)\right\|_{p} \\
& \leq\left(\sum_{\ell=n}^{m-1} \frac{C^{(4)}}{\ell !}\left(C^{(3)}(t-T)\right)^{\ell}\right) I(t-T) .
\end{aligned}
$$

Thus, $\rho^{(k)}$ is Cauchy with respect to the norm

$$
\|\sigma\|=\sup _{t \in[0, T+\delta]}\|\sigma(t)\|_{p}
$$

Using this estimate with (77), we may conclude that $E^{(k)}$ is Cauchy in $L^{\infty}([0, T+\delta] \times \mathbb{R})$. Define $X^{(k)}(s, t, x, v)$ and $V^{(k)}(s, t, x, v)$ by

$$
\begin{cases}\frac{d X^{(k)}}{d s}=V^{(k)}, & X^{(k)}(t, t, x, v)=x \\ \frac{d V^{(k)}}{d s},=-\left.E^{(k)}\right|_{\left(s, X^{(k)}(s)\right)} & V^{(k)}(t, t, x, v)=v .\end{cases}
$$

Then, since $f^{(n)}, f^{(m)} \in \mathcal{C}$, we use the Mean Value Theorem to find

$$
\begin{aligned}
\left|\left(X^{(n)}-X^{(m)}\right)(s)\right|+\left|\left(V^{(n)}-V^{(m)}\right)(s)\right| \leq & \int_{s}^{t}\left(\left|\left(V^{(n)}-V^{(m)}\right)(\tau)\right|\right. \\
& +\left|E^{(n)}\left(\tau, X^{(n)}(\tau)\right)-E^{(m)}\left(\tau, X^{(m)}(\tau)\right)\right| d \tau \\
\leq & \int_{s}^{t}\left(\mid\left(V^{(n)}-V^{(m)}(\tau) \mid\right.\right. \\
& +\left|E^{(n)}\left(\tau, X^{(n)}(\tau)\right)-E^{(n)}\left(\tau, X^{(m)}(\tau)\right)\right| \\
& \left.+\left|E^{(n)}\left(\tau, X^{(m)}(\tau)\right)-E^{(m)}\left(\tau, X^{(m)}(\tau)\right)\right|\right) d \tau \\
\leq & \int_{s}^{t}\left(\mid\left(V^{(n)}-V^{(m)}(\tau) \mid\right.\right. \\
& \left.+\left\|\rho^{(n)}(\tau)\right\|_{p}\left|\left(X^{(n)}-X^{(m)}\right)(\tau)\right|\right) d \tau \\
& +\left\|E^{(n)}-E^{(m)}\right\|_{L^{\infty}([0, T+\delta] \times \mathbb{R})} \\
\leq & \left\|E^{(n)}-E^{(m)}\right\|_{L^{\infty}([0, T+\delta] \times \mathbb{R})} \\
& +C \int_{s}^{t}\left(\left|\left(X^{(n)}-X^{(m)}\right)(\tau)\right|+\mid\left(V^{(n)}-V^{(m)}(\tau) \mid\right) d \tau\right.
\end{aligned}
$$


Using Gronwall's Inequality, we have for $0 \leq s \leq t \leq T+\delta$

$$
\left|\left(X^{(n)}-X^{(m)}\right)(s)\right|+\left|\left(V^{(n)}-V^{(m)}\right)(s)\right| \leq C\left\|E^{(n)}-E^{(m)}\right\|_{L^{\infty}([0, T+\delta] \times \mathbb{R})} .
$$

Thus, $X^{(k)}$ and $V^{(k)}$ are uniformly Cauchy. Then, by definition of the iterates,

$$
f^{(k+1)}(t, x, v)=f_{0}\left(X^{(k)}(0, t, x, v), V^{(k)}(0, t, x, v)\right) .
$$

So, define

$$
f(t, x, v)=\lim _{k \rightarrow \infty} f_{0}\left(X^{(k)}(0, t, x, v), V^{(k)}(0, t, x, v)\right) .
$$

Then, since $f^{(k)} \in \mathcal{C}$ and thus $\left\|\rho^{(k)}(t)\right\|_{p} \leq C$, we use (A-3), Lemma 1, and the Bounded Convergence Theorem to define

$$
\rho=\lim _{k \rightarrow \infty} \rho^{(k)}=\lim _{k \rightarrow \infty} \int\left(F-f^{(k)}\right) d v=\int(F-f) d v
$$

Similarly, define

$$
E=\lim _{k \rightarrow \infty} E^{(k)}=\lim _{k \rightarrow \infty} \frac{1}{2}\left(\int_{-\infty}^{x} \rho^{(k)}(t, y) d y-\int_{x}^{\infty} \rho^{(k)}(t, y) d y\right)
$$

and use Lebesgue's Dominated Convergence Theorem to conclude that (4) holds. Define

$$
X=\lim _{k \rightarrow \infty} X^{(k)}
$$

and

$$
V=\lim _{k \rightarrow \infty} V^{(k)} .
$$

It follows from (26) and the field bound that (9) holds. Also, from (27), we have

$$
f(t, x, v)=f_{0}(X(0, t, x, v), V(0, t, x, v))
$$

and thus

$$
f(t, x, v)=f(s, X(s, t, x, v), V(s, t, x, v))
$$

for all $s \in[0, t]$. Finally, $f^{(k)} \in C$ for each $k$ implies $\rho$ is Lipschitz in $x$ and thus $E$ is $C^{1}$ in $x$. We may use (5) and Lemma 1 to conclude that $E$ is continuous in $t$. Thus, we see that $X$ and $V$ are $C^{1}$ from (9), $f$ is $C^{1}$ from (28), and $f$ satisfies the Vlasov equation from (29).

In order to show uniqueness, we let $f, h \in \mathcal{C}$ be given solutions with $f(0, x, v)=h(0, x, v)=$ $f_{0}(x, v)$ for all $x, v \in \mathbb{R}$. Then, since $f, h \in \mathcal{C}$ solve (11) we use (25) to conclude

$$
\left\|\left(\rho-\rho_{h}\right)(t)\right\|_{p} \leq C(t-T) I(t-T) .
$$

We may repeatedly apply (25) and use induction to find for every $k \in \mathbb{N}$

$$
\left\|\left(\rho-\rho_{h}\right)(t)\right\|_{p} \leq \frac{C^{(4)}}{k !}\left(C^{(3)}(t-T)\right)^{k} I(t-T) .
$$

This implies

$$
\left\|\left(\rho-\rho_{h}\right)(t)\right\|_{p}=0 .
$$

Therefore, $\rho \equiv \rho_{h}, E \equiv E_{h}$ and by uniqueness of characteristics, $f \equiv h$. Thus, the proofs of Theorem 1 and Theorem 2 are complete. 


\section{Section 3}

The section which follows is devoted to the proof of Lemmas 1, 2, 3, and 4 .

Proof of Lemma 1: Using (8) and (91), we may conclude

$$
\begin{aligned}
|V(s, t, x, v)| & =\left|v+\int_{s}^{t} E(\tau, X(\tau, t, x, v)) d \tau\right| \\
& \geq|v|-\int_{0}^{t}\|E(\tau)\|_{\infty} d \tau \\
& \geq|v|-C^{(1)} .
\end{aligned}
$$

Similarly, we find

$$
|V(s, t, x, v)| \leq|v|+C^{(1)} .
$$

Thus, for $|v|>2 C^{(1)}$, we have

$$
\frac{1}{2}|v| \leq|v|-C^{(1)} \leq|V(s, t, x, v)| \leq|v|+C^{(1)} \leq \frac{3}{2}|v|
$$

and the proof of Lemma 1 is complete.

Proof of Lemma 2 : Let $\mathcal{E} \in \mathcal{C}^{1}(\mathbb{R}), H \in \mathcal{C}_{c}^{2}(\mathbb{R})$, and $B>0$ be given with

$$
|\mathcal{E}(x)| \leq B
$$

and

$$
\left|\mathcal{E}^{\prime}(x)\right| \leq B R^{-p}(x)
$$

for all $x \in \mathbb{R}$. Let $t \in[0, T+\delta]$ and $s \in[0, t]$ be given. Since $H$ has compact support, let $\tilde{W}:=\sup \{|v|: H(v) \neq 0\}$ and define $C^{(5)}:=2 \max \left\{C^{(1)}, \tilde{W}\right\}$. Then, using Lemma 1, we find for $|v|>C^{(5)}$ that $|V(s)| \geq \frac{1}{2}|v| \geq \tilde{W}$, and thus $H^{\prime}(V(s))=0$. Define

$$
C^{(6)}:=(T+\delta)\left(C^{(1)}+C^{(5)}\right) .
$$

We have for $|x| \leq 2 C^{(6)}$

$$
\begin{aligned}
\left|\int \mathcal{E}(X(s, t, x, v)) H^{\prime}(V(s, t, x, v)) d v\right| & \leq \int_{|v| \leq C^{(5)}} B\left\|H^{\prime}\right\|_{\infty} d v \\
& \leq C B \leq C B R^{-p}(x) .
\end{aligned}
$$

Take $|x|>2 C^{(6)}$ and write 


$$
\begin{aligned}
& \int \mathcal{E}(X(s, t, x, v)) H^{\prime}(V(s, t, x, v)) d v \\
= & \int_{|v| \leq C(5)}\left[\mathcal{E}(X(s, t, x, v))\left(H^{\prime}(V(s, t, x, v))-H^{\prime}\left(v+\int_{s}^{t} E(\tau, x) d \tau\right)\right)\right. \\
& +(\mathcal{E}(X(s, t, x, v))-\mathcal{E}(x+(s-t) v)) H^{\prime}\left(v+\int_{s}^{t} E(\tau, x) d \tau\right) \\
& +\frac{d}{d v}\left(H\left(v+\int_{s}^{t} E(\tau, x) d \tau\right) \mathcal{E}(x+(s-t) v)\right) \\
& \left.-H\left(v+\int_{s}^{t} E(\tau, x) d \tau\right) \frac{d}{d v}(\mathcal{E}(x+(s-t) v))\right] d v \\
=: & I+I I+I I I+I V .
\end{aligned}
$$

To estimate $I$, we use the Mean Value Theorem to find $\xi_{1}$ between $x$ and $X(\tau)$ such that

$$
E(\tau, X(\tau))-E(\tau, x)=\partial_{x} E\left(\tau, \xi_{1}\right)(X(\tau)-x) .
$$

In addition, notice that for $|v| \leq C^{(5)}$, we have using Lemma 1

$$
\begin{aligned}
\left|\xi_{1}\right| & \geq|x|-|X(\tau)-x| \\
& \geq|x|-\left(\int_{\tau}^{t}\left(|v|+C^{(1)}\right) d s\right) \\
& \geq|x|-(T+\delta)\left(C^{(5)}+C^{(1)}\right) \\
& \geq|x|-C^{(6)} \\
& \geq \frac{1}{2}|x| .
\end{aligned}
$$

Using (6), (30), and Lemma 1 we find

$$
\begin{aligned}
I & \leq C B \int_{|v| \leq C^{(5)}}\left|H^{\prime}(V(s, t, x, v))-H^{\prime}\left(v+\int_{s}^{t} E(\tau, x) d \tau\right)\right| d v \\
& \leq C B\left\|H^{\prime \prime}\right\|_{\infty} \int_{|v| \leq C^{(5)}} \int_{s}^{t}|E(\tau, X(\tau))-E(\tau, x)| d \tau d v \\
& \leq C B \int_{|v| \leq C^{(5)}} \int_{s}^{t}\left|\partial_{x} E\left(\tau, \xi_{1}\right)\right||X(\tau)-x| d \tau d v \\
& \leq C B \int_{|v| \leq C^{(5)}} \int_{s}^{t}\|\rho(\tau)\|_{p} R^{-p}\left(\xi_{1}\right)\left(\int_{\tau}^{t}|V(\lambda)| d \lambda\right) d \tau d v \\
& \leq C B R^{-p}\left(\frac{1}{2}|x|\right)\left(\int_{|v| \leq C^{(5)}}\left(|v|+C^{(1)}\right) d v\right)\left(\int_{s}^{t}(1+r I(\tau-T)) d \tau\right) \\
& \leq C B R^{-p}(x) .
\end{aligned}
$$

To estimate $I I$, we again use the Mean Value Theorem and find $\xi_{2}$ between $X(s)$ and $x+(s-t) v$ such that

$$
\mathcal{E}(X(s))-\mathcal{E}(x+(s-t) v)=\mathcal{E}^{\prime}\left(\xi_{2}\right)(X(s)-(x+(s-t) v)) .
$$


In addition, notice that for $|v| \leq C^{(5)}$, we use Lemma 1 and (을 to find

$$
\begin{aligned}
\left|\xi_{2}\right| & \geq|x+(s-t) v|-|X(s)-(x+(s-t) v)| \\
& \geq|x|-(T+\delta) C^{(5)}-\left|\int_{s}^{t} \int_{\tau}^{t} E(\iota, X(\iota)) d \iota d \tau\right| \\
& \geq|x|-(T+\delta)\left(C^{(5)}+C^{(1)}\right) \\
& \geq \frac{1}{2}|x| .
\end{aligned}
$$

Then, using (A-2), (8), and (31), we find

$$
\begin{aligned}
I I & \leq C\left\|H^{\prime}\right\|_{\infty} \int_{|v| \leq C^{(5)}}|\mathcal{E}(X(s, t, x, v))-\mathcal{E}(x+(s-t) v)| d v \\
& \leq C \int_{|v| \leq C^{(5)}}\left|\mathcal{E}^{\prime}\left(\xi_{2}\right)\right||X(s)-x-(s-t) v| d v \\
& \leq C B \int_{|v| \leq C^{(5)}} R^{-p}\left(\xi_{2}\right) \int_{s}^{t} \int_{\tau}^{t}|E(\iota, X(\iota))| d \iota d \tau \\
& \leq C B R^{-p}(x) .
\end{aligned}
$$

By the Fundamental Theorem of Calculus and compact support of $H$, we find

$$
I I I=0 .
$$

To estimate $I V$, notice that for $|v| \leq C^{(5)}$,

$$
|x+(s-t) v| \geq|x|-(T+\delta) C^{(5)} \geq|x|-C^{(6)} \geq \frac{1}{2}|x| .
$$

Thus, we use (31), (32), and Lemma 1 to estimate $I V$, which yields

$$
\begin{aligned}
I V & \leq C\|H\|_{\infty} \int_{|v| \leq C^{(5)}}|(s-t)|\left|\mathcal{E}^{\prime}(x+(s-t) v)\right| d v \\
& \leq C B \int_{|v| \leq C^{(5)}} R^{-p}(x+(s-t) v) d v \\
& \leq C B R^{-p}(x) .
\end{aligned}
$$

Combining the estimates for $I-I V$, we have

$$
\mid \int \mathcal{E}\left(X(s) H^{\prime}(V(s)) d v \mid \leq C B R^{-p}(x)\right.
$$

for $|x|>2 C^{(6)}$, and the lemma follows.

Proof of Lemma 3 : We must bound $Q_{\tilde{g}}(t)$. Define for $t \in[0, T+\delta]$,

$$
Q(t):=\sup \{|v|: \exists x \in \mathbb{R}, \tau \in[0, t] \text { s.t. } \tilde{f}(\tau, x, v) \neq 0\} .
$$

By (A-1), we know that $Q(0)$ is finite. By the definition of $Q(t)$, if $|V(0, t, x, v)| \geq Q(0)$, we have for every $y \in \mathbb{R}$,

$$
f_{0}(y, V(0, t, x, v))=0
$$


But, by Lemma 1, if $|v| \geq 2 \max \left\{Q(0), C^{(1)}\right\}$, then

$$
|V(0, t, x, v)| \geq \frac{1}{2}|v| \geq Q(0)
$$

which implies that $f_{0}(y, V(0, t, x, v))=0$. So, if $f_{0}(y, V(0, t, x, v)) \neq 0$ for some $y \in \mathbb{R}$, we must have

$$
|v| \leq 2 \max \left\{Q(0), C^{(1)}\right\} .
$$

The definition of $\tilde{f}$ implies that $\tilde{f}(t, x, v)=f_{0}(X(0, t, x, v), V(0, t, x, v))$. So, if $\tilde{f}(t, x, v) \neq 0$ for some $t \in[0, T+\delta], x, v \in \mathbb{R}$, then (33) must hold. Taking the supremum over $v$ of both sides in (33), we find

$$
Q(t) \leq 2 \max \left\{Q(0), C^{(1)}\right\} \leq C
$$

for every $t \in[0, T+\delta]$. Since $F$ is compactly supported and $Q_{\tilde{g}}(t)=\max \{Q(t), W\}$, it follows that

$$
Q_{\tilde{g}}(t) \leq C
$$

for all $t \in[0, T+\delta]$, as well.

Proof of Lemma 4: Let $t \in[0, T+\delta], s \in[0, t]$, and $x \in \mathbb{R}$ be given. Notice that by the definitions of $E$ and $E_{h}$, we have

$$
\partial_{x}\left(E-E_{h}\right)(t, x)=\left(\rho-\rho_{h}\right)(t, x) .
$$

In addition, using (6) and (17), we have

$$
\left|\left(E-E_{h}\right)(t, x)\right| \leq C\left\|\left(\rho-\rho_{h}\right)(t)\right\|_{p}
$$

and

$$
\left|\partial_{x}\left(E-E_{h}\right)(t, x)\right| \leq C\left\|\left(\rho-\rho_{h}\right)(t)\right\|_{p} R^{-p}(x) .
$$

Define $C^{(7)}:=(T+\delta)\left(Q_{\tilde{g}_{h}}(T+\delta)+C^{(1)}\right)$. Then, for $|x| \leq 2 C^{(7)}$, we use (21) and (35) to find

$$
\begin{aligned}
\left|\int\left(E-E_{h}\right)(s, X(s)) \partial_{v} \tilde{g}_{h}(s, X(s), V(s)) d v\right| & \leq C \int_{|v| \leq Q_{\tilde{g}_{h}}(s)}\left\|\left(\rho-\rho_{h}\right)(s)\right\|_{p} d v \\
& \leq C\left\|\left(\rho-\rho_{h}\right)(s)\right\|_{p} \\
& \leq C\left\|\left(\rho-\rho_{h}\right)(s)\right\|_{p} R^{-p}(x) .
\end{aligned}
$$

Now, let $|x|>2 C^{(7)}$, and notice that for $|v| \leq Q_{\tilde{g}_{h}}(s)$ and $\tau \in[0, s]$, we have

$$
\begin{aligned}
|X(\tau)| & \geq|x|-\int_{\tau}^{t}|V(\iota)| d \iota \\
& \geq|x|-\int_{\tau}^{t}\left(|v|+C^{(1)}\right) d \iota \\
& \geq|x|-(T+\delta)\left(Q_{\tilde{g}_{h}}(T+\delta)+C^{(1)}\right) \\
& \geq|x|-C^{(7)} .
\end{aligned}
$$

Thus, for $|v| \leq Q_{\tilde{g}_{h}}(s)$ and $\tau \in[0, s]$, we have

$$
|X(\tau)| \geq \frac{1}{2}|x| .
$$


Integrating along characteristics in (15), we find

$$
\left.\partial_{v} \tilde{g}_{h}\right|_{(s, X(s), V(s))}=\left.\partial_{v} \tilde{g}_{h}\right|_{(0, X(0), V(0))}-\left.\int_{0}^{s}\left(E_{h} F^{\prime \prime}+\partial_{x} \tilde{g}_{h}\right)\right|_{(\tau, X(\tau), V(\tau))} d \tau .
$$

Using this, we may write

$$
\begin{aligned}
\left.\int\left[\left(E-E_{h}\right) \partial_{v} \tilde{g}_{h}\right]\right|_{(s, X(s), V(s))} d v & =\left.\int_{|v| \leq Q_{\tilde{g}_{h}}(s)}\left(E-E_{h}\right)\right|_{(s, X(s), V(s))} \\
& \cdot\left[\left.\partial_{v} \tilde{g}_{h}\right|_{(0, X(0), V(0))}-\left.\int_{0}^{s}\left(E_{h} F^{\prime \prime}+\partial_{x} \tilde{g}_{h}\right)\right|_{(\tau, X(\tau), V(\tau))} d \tau\right] d v \\
\leq & I+I I+I I I
\end{aligned}
$$

where

$$
\begin{gathered}
I=\left|\int_{|v| \leq Q_{\tilde{g}_{h}}(s)}\left(E-E_{h}\right)(s, X(s)) \partial_{v} \tilde{g}_{h}(0, X(0), V(0)) d v\right|, \\
I I=\left|\int_{|v| \leq Q_{\tilde{g}_{h}}(s)}\left(E-E_{h}\right)(s, X(s)) \int_{0}^{s} E_{h}(\tau, X(\tau)) F^{\prime \prime}(V(\tau)) d \tau d v\right|,
\end{gathered}
$$

and

$$
I I I=\left|\int_{|v| \leq Q_{\tilde{g}_{h}}(s)}\left(E-E_{h}\right)(s, X(s))\left(\int_{0}^{s} \partial_{x} \tilde{g}_{h}(\tau, X(\tau), V(\tau)) d \tau\right) d v\right| .
$$

To estimate $I$, we use (A-1), (35) and (37) to find

$$
\begin{aligned}
I & \leq C\left\|\left(\rho-\rho_{h}\right)(s)\right\|_{p} \int_{|v| \leq Q_{\tilde{g}_{h}}(s)} R^{-p}(X(0)) d v \\
& \leq C\left\|\left(\rho-\rho_{h}\right)(s)\right\|_{p} Q_{\tilde{g}_{h}}(s) R^{-p}\left(\frac{1}{2}|x|\right) \\
& \leq C\left\|\left(\rho-\rho_{h}\right)(s)\right\|_{p} R^{-p}(x) .
\end{aligned}
$$

To estimate $I I$, we write

$$
\begin{aligned}
\left(E-E_{h}\right)(s, X(s)) E_{h}(\tau, X(\tau)) F^{\prime \prime}(V(\tau))= & \left(\left(E-E_{h}\right)(s, X(s))-\left(E-E_{h}\right)(s, x)\right) E_{h}(\tau, X(\tau)) F^{\prime \prime}(V(\tau)) \\
& +\left(E-E_{h}\right)(s, x) E_{h}(\tau, X(\tau)) F^{\prime \prime}(V(\tau)) .
\end{aligned}
$$

For the first term in (38), we use the Mean Value Theorem and find $\xi_{3}$ between $X(s)$ and $x$ such that

$$
\left(E-E_{h}\right)(s, X(s))-\left(E-E_{h}\right)(s, x)=\partial_{x}\left(E-E_{h}\right)\left(s, \xi_{3}\right)(X(s)-x) .
$$

In addition, notice that for $|v| \leq Q_{\tilde{g}_{h}}(s)$, we use Lemma 1 and (8) to find

$$
\begin{aligned}
\left|\xi_{3}\right| & \geq|x|-|X(s)-x| \\
& \geq|x|-\int_{s}^{t}|V(\iota)| d \iota \\
& \geq|x|-(T+\delta)\left(Q_{\tilde{g}_{h}}(T+\delta)+C^{(1)}\right) \\
& \geq|x|-C^{(7)} \\
& \geq \frac{1}{2}|x| .
\end{aligned}
$$


Thus, using (7) and (36), we find

$$
\begin{aligned}
& \mid \int_{|v| \leq Q_{\tilde{g}_{h}}(s)}\left(\left(E-E_{h}\right)(s, X(s))-\left(E-E_{h}(s, x)\right) E_{h}(\tau, X(\tau)) F^{\prime \prime}(V(\tau)) d v \mid\right. \\
\leq & \int_{|v| \leq Q_{\tilde{g}_{h}}(s)}\left|\partial_{x}\left(E-E_{h}\right)\left(s, \xi_{3}\right)(X(s)-x)\right|\left|E_{h}(\tau, X(\tau)) F^{\prime \prime}(V(\tau))\right| d v \\
\leq & C\left(Q_{\tilde{g}_{h}}(s)+C^{(1)}\right)(T+\delta)\left\|F^{\prime \prime}\right\|_{L^{\infty}(\mathbb{R})}\|\rho(\tau)\|_{p}\left\|\left(\rho-\rho_{h}\right)(s)\right\|_{p} R^{-p}\left(\xi_{3}\right) \\
\leq & C\|\rho(\tau)\|_{p}\left\|\left(\rho-\rho_{h}\right)(s)\right\|_{p} R^{-p}(x)
\end{aligned}
$$

and therefore,

$$
\mid \int_{|v| \leq Q_{\tilde{g}_{h}}(s)}\left(\left(E-E_{h}\right)(s, X(s))-\left(E-E_{h}(s, x)\right) E_{h}(\tau, X(\tau)) F^{\prime \prime}(V(\tau)) d v \mid \leq C\|\rho(\tau)\|_{p}\left\|\left(\rho-\rho_{h}\right)(s)\right\|_{p} R^{-p}(x) .\right.
$$

Then, to estimate the second term of (38) we use (7), (35), and (36) in Lemma 2 (with $\mathcal{E}=$ $\left(E-E_{h}\right)(s, x) E_{h}(\tau, X(\tau))$ and $\left.H=F^{\prime}\right)$ to find

$$
\left|\int_{|v| \leq Q_{\tilde{g}_{h}}(s)}\left(E-E_{h}\right)(s, x) E_{h}(\tau, X(\tau)) F^{\prime \prime}(V(\tau)) d v\right| \leq C\left\|\rho_{h}(\tau)\right\|_{p}\left\|\left(\rho-\rho_{h}\right)(s)\right\|_{p} R^{-p}(x) .
$$

Finally, using (6), (39), and (40), we find

$$
\begin{aligned}
I I= & \left|\int_{0}^{s} \int_{|v| \leq Q_{\tilde{g}_{h}}(s)}\left(E-E_{h}\right)(s, X(s)) E_{h}(\tau, X(\tau)) F^{\prime \prime}(V(\tau)) d v d \tau\right| \\
\leq & \int_{0}^{s}\left(\left|\int_{|v| \leq Q_{\tilde{g}_{h}}(s)}\left(\left(E-E_{h}\right)(s, X(s))-\left(E-E_{h}\right)(s, x)\right) E_{h}(\tau, X(\tau)) F^{\prime \prime}(V(\tau)) d v\right|\right. \\
& \left.+\left|\int_{|v| \leq Q_{\tilde{g}_{h}}(s)}\left(E-E_{h}\right)(s, x) E_{h}(\tau, X(\tau)) F^{\prime \prime}(V(\tau)) d v\right|\right) d \tau \\
\leq & C\left\|\left(\rho-\rho_{h}\right)(s)\right\|_{p}\left(\int_{0}^{s}\left\|\rho_{h}(\tau)\right\|_{p} d \tau\right) R^{-p}(x) \\
\leq & C\left\|\left(\rho-\rho_{h}\right)(s)\right\|_{p} R^{-p}(x)\left(\int_{0}^{s}(1+r I(\tau-T)) d \tau\right) \\
\leq & C\left\|\left(\rho-\rho_{h}\right)(s)\right\|_{p} R^{-p}(x) .
\end{aligned}
$$

To estimate $I I I$, we use (21), (35), and (37) to find

$$
\begin{aligned}
I I I & \leq C \int_{|v| \leq Q_{\tilde{g}_{h}}(s)}\left\|\left(\rho-\rho_{h}\right)(s)\right\|_{p}\left(\int_{0}^{s}\left\|\partial_{x} \tilde{g}_{h}(\tau)\right\|_{p} R^{-p}(X(\tau)) d \tau\right) d v \\
& \leq C Q_{\tilde{g}_{h}}(s)\left(\int_{0}^{s}\left\|\partial_{x} \tilde{g}_{h}(\tau)\right\|_{p} d \tau\right)\left\|\left(\rho-\rho_{h}\right)(s)\right\|_{p} R^{-p}\left(\frac{1}{2}|x|\right) \\
& \leq C\left\|\left(\rho-\rho_{h}\right)(s)\right\|_{p} R^{-p}(x)
\end{aligned}
$$

Combining the estimates for $I, I I$, and $I I I$, the lemma follows and the proof is complete. 


\section{References}

[1] Batt, J. Global symmetric solutions of the initial-value problem of stellar dynamics. J. Diff. Eq. 1977, 25:342-364.

[2] Batt, J.; Kunze, M.; and Rein, G. On the asymptotic behavior of a one-dimensional, monocharged plasma and a rescaling method. Advances in Differential Equations 1998, 3:271292.

[3] Burgan, J.R.; Feix, M.R.; Fijalkow, E.; Munier, A. Self-similar and asymptotic solutions for a one-dimensional Vlasov beam. J. Plasma Physics 1983, 29:139-142.

[4] Glassey, R. The Cauchy Problem in Kinetic Theory; S.I.A.M: Philadelphia, 1996.

[5] Horst, E. On the classical solutions of the initial value problem for the unmodified nonlinear Vlasov-equation, Parts I and II. Math. Methods Appl. Sci. 1981, 3:229-248 and 1982, 4:19-32.

[6] Lions, P.L.; Pertham, B. Propogation of moments and regularity for the three dimensional Vlasov-Poisson system. Invent. Math. 1991, 105:415-430.

[7] Pankavich, S. Global existence for the Radial Vlasov-Possion System with Steady Spatial Asymptotics. 2004 (submitted for publication).

[8] Pankavich, S. Global existence for the Vlasov-Possion System with Steady Spatial Asymptotics. Comm. PDE 2006, 31:249-270.

[9] Pfaffelmoser, K. Global classical solution of the Vlassov-Poisson system in three dimensions for general initial data. J. Diff. Eq. 1992, 95(2):281-303.

[10] Rein, G. Collisionless Kinetic Equations from Astrophysics-The Vlasov-Poisson System. Unpublished manuscript, 2005.

[11] Schaeffer, J. Global existence of smooth solutions to the Vlasov-Poisson system in three dimensions. Comm. PDE. 1991, 16(8/9):1313-1335.

[12] Schaeffer, J. Steady spatial asymptotics for the Vlasov-Poisson system. Math. Methods Appl. Sci. 2003, 26:273-296.

[13] Schaeffer, J. The Vlasov-Poisson system with steady spatial asymptotics. Comm. PDE. 2003, 28(5/6):1057-1084. 